\title{
HELICAL DIPOLE MAGNETS FOR POLARIZED PROTONS IN RHIC
}

M. Syphers ${ }^{\dagger}$, E. Courant, W. Fischer, A. Luccio, F. Mariam, S. Peggs, F. Pilat, T. Roser,

S. Tepikian, N. Tsoupas, E. Willen, Brookhaven National Laboratory, Upton, NY, USA,

T. Katayama, U. of Tokyo, Japan, K. Hatanaka, Osaka U., Japan,

RECEIVED

T. Kawaguchi, M. Okamura, T. Tominaka, H. Wu, RIKEN, Saitama, Japan, and

V. Ptitsin, Y. Shatunov, BINP, Novosibirsk, Russia

\section{Abstract}

Superconducting helical dipole magnets will be used in the Brookhaven Relativistic Heavy Ion Collider (RHIC) to maintain polarization of proton beams and to perform localized spin rotations at the two major experimental detector regions. Requirements for the helical dipole system are discussed, and magnet prototype work is reported.

\section{SNAKES AND SPIN ROTATORS IN RHIC}

The Relativistic Heavy Ion Collider at Brookhaven National Laboratory allows for the unique possibility of colliding high energy polarized proton beams. To maintain polarization during the acceleration process, two full "Siberian Snakes" are to be inserted on opposite sides of the RHIC lattice for each of the two counter-rotating rings. In addition, other magnetic components -- spin rotators -- will be located on each side of the two major interaction points (again, for each ring) which allow the spin orientation to be altered from the vertical direction to the longitudinal direction. Superconducting magnets are used in order to contain the magnetic elements for a Snake within a $10 \mathrm{~m}$ longitudinal space so as to fit within available room in the RHIC lattice. The use of a helical dipole field as part of a Siberian Snake in a synchrotron was first suggested by Courant[1]. A system made completely of four identical length helices was first proposed by Ptitsin and Shatunov[2]. Four right-handed helical dipole magnets, each $2.4 \mathrm{~m}$ long and operating near $4 \mathrm{~T}$ or less can produce a Siberian Snake for RHIC. The strong helical fields reduce the orbit excursions normally produced by interleaved horizontal and vertical dipole magnets. Hence, the magnet apertures can be similar to those found elsewhere in RHIC. Furthermore, a combination of right-handed and lefthanded helical dipole magnets also within a $10 \mathrm{~m}$ space can perform the desired local $90^{\circ}$ rotations of the spin at the major detector regions.

\subsection{Helical Magnets for Spin Control}

Let $x$ and $y$ be the transverse coordinates, $z$ the longitudinal coordinate, and $k \equiv 2 \pi / L$, with $L$ the repeat period of the helix. Then the magnetic field of a helical dipole magnet in which the field begins in the vertical direction (at $z=0$ ) can be described to lowest order in Cartesian coordinates according to[3]

$$
\begin{aligned}
& B_{x}=-B_{0}\left\{\left[1+\frac{k^{2}}{8}\left(3 x^{2}+y^{2}\right)\right] \sin k z-\frac{k^{2}}{4} x y \cos k\right\}, \\
& B_{y}=B_{0}\left\{\left[1+\frac{k^{2}}{8}\left(x^{2}+3 y^{2}\right)\right] \cos k-\frac{k^{2}}{4} x y \sin k\right\}, \\
& B_{z}=-B_{0} k\left\{1+\frac{k^{2}}{8}\left(x^{2}+y^{2}\right)\right\}[x \cos k+y \sin k z\}
\end{aligned}
$$

The twisting current distribution introduces intrinsic nonlinear terms into the field. To first order, the trajectory through the helical field above is just

$$
\begin{aligned}
& x(z)=x_{0}-\frac{B_{0}}{(B \rho)} \frac{1}{k^{2}}(1-\cos k z)+x_{0}^{\prime} z, \\
& y(z)=y_{0}+\frac{B_{0}}{(B \rho)} \frac{1}{k^{2}} \sin k z+\left(y_{0}^{\prime}-\frac{B_{0}}{(B \rho)} \frac{1}{k}\right) z .
\end{aligned}
$$

We see that for a complete $360^{\circ}$ helix, the effect on the particle trajectory is simply a vertical "shift" in the orbit by an amount $\delta=-B_{0} L k(B \rho)$. We also note that the handedness of the helix is given by the sign of $k$, positive is right-handed, negative is left-handed. By pairing identical length $360^{\circ}$ helical magnets of the same strength but opposite field directions (or, equivalently, with equal strength but opposite handedness), the overall orbit distortion outside the system will be zero. With four magnets, this allows one to choose two independent field strengths for control of the spin rotation angle and axis of rotation. A third parameter is needed to completely define the rotation, but by providing the system with reflection symmetry, the axis of rotation is constrained to lie in the horizontal plane.

The spin precession through a helical dipole magnet is most easily found by using the spinor formalism and solving the equation of motion for the first-order field expressions[4],[5]. For the field description given above the axis of rotation, $n$, and angle of rotation, $\mu$, can be written as

$$
\mathrm{n}=\frac{\mathrm{x}_{1}+\left(\frac{\mathrm{x}}{k}\right) \mathrm{x}_{3}}{\sqrt{1+\left(\frac{\mathrm{x}}{k}\right)^{2}}} \quad \text { and } \quad \mu=-2 \pi \sqrt{1+\left(\frac{x}{k}\right)^{2}}
$$

Tork performed under the auspices of the U.S. Department of Energy and partially supported by The Institute for Chemical and Physical Research (RIKEN), Japan. 
where $k=(1+G \gamma) B_{\alpha} /(B \rho)$, and $x_{b}, x_{3}$ are unit vectors in the longitudinal and vertical directions, respectively. With Eqs. 4-6, the first-order orbit and spin behavior through a system of helical dipole magnets can be readily studied and optimized.

\subsection{RHIC Helical Magnet System}

In the present RHIC design Snake helical dipoles are all $360^{\circ}$ right-handed helices whose fields begin pointed vertically upward or downward. The "Rotator" magnets are either left-handed or right-handed, but each begins with its field pointed in the horizontal plane.

\begin{tabular}{||c|c|c|c|}
\hline Snake & \multicolumn{2}{|c|}{ inj. orbit dev: } & \multicolumn{2}{c|}{$32 \mathrm{~mm}$} & \\
\hline Length & start/end & helicity & max. field \\
\hline $2.4 \mathrm{~m}$ & vertical & $\mathrm{RH}$ & $1.2 \mathrm{~T}$ \\
$2.4 \mathrm{~m}$ & vertical & $\mathrm{RH}$ & $-3.9 \mathrm{~T}$ \\
$2.4 \mathrm{~m}$ & vertical & $\mathrm{RH}$ & $3.9 \mathrm{~T}$ \\
$2.4 \mathrm{~m}$ & vertical & $\mathrm{RH}$ & $-1.2 \mathrm{~T}$ \\
\hline Rotator & inj. orbit dev: & $24 \mathrm{~mm}$ & \\
\hline Length & startend & helicity & max. field \\
\hline $2.4 \mathrm{~m}$ & horizontal & $\mathrm{RH}$ & $3.4 \mathrm{~T}$ \\
$2.4 \mathrm{~m}$ & horizontal & $\mathrm{LH}$ & $3.1 \mathrm{~T}$ \\
$2.4 \mathrm{~m}$ & horizontal & $\mathrm{RH}$ & $3.1 \mathrm{~T}$ \\
$2.4 \mathrm{~m}$ & horizontal & $\mathrm{LH}$ & $3.4 \mathrm{~T}$ \\
\hline
\end{tabular}

Table 1: Helical Magnet Parameters for Siberian Snakes and Spin Rotators in RHIC.

The field strengths of the Snake magnets are kept constant during the acceleration process, while the appropriate fields in the Rotator magnets are beam energy dependent. This is due to the fact that there is a net horizontal bending of $\theta=3.7 \mathrm{mrad}$ between the spin rotator location and the interaction point. Hence once the spin vector is rotated into the horizontal plane by the spin Rotator system, it will undergo further precession of $G \gamma \theta$ about the vertical by the time it reaches the interaction point.

Table 1 shows the magnet parameters of the system. The Rotator fields are for $250 \mathrm{GeV}$ proton operation. The maximum orbit deviations listed in each case, however, are for an injection energy of 25 $\mathrm{GeV}$. The magnet fields, particle trajectories, and spin precession for a Siberian Snake are shown in Figure 1. Figure 2 shows a plot of the required values of the two independent magnetic fields in the spin Rotators for various RHIC energies[6].

The effects on RHIC operation of helical dipole magnet error fields and misalignments have been studied. In contrast to a "regular" dipole magnet error which can be thought of as producing a kink in the slope of the particle trajectory at the source of the error, a "helical" dipole error will introduce a step in the trajectory. To keep the vertical orbit distortions under control, the helical dipole field errors $\triangle B L(B L)$ should be kept reasonably below $1 \%$, and rotational misalignments should be less than about $10 \mathrm{mrad}[7]$. Another important parameter will be the total integrated field strength ( $\int B_{x} d s, \int B_{y} d s$ ) which should be zero, or equivalently the total effective integrated twist of the magnet should be $360^{\circ}$. With careful orbit correction in the vicinity of the helical dipoles, it is expected that this effective twist angle should be $360^{\circ}$ $\pm 2^{\circ}$ [8]. The ends of the magnets will need to be carefully designed to obtain not only the desired integrated field strength but also the desired total field twist.
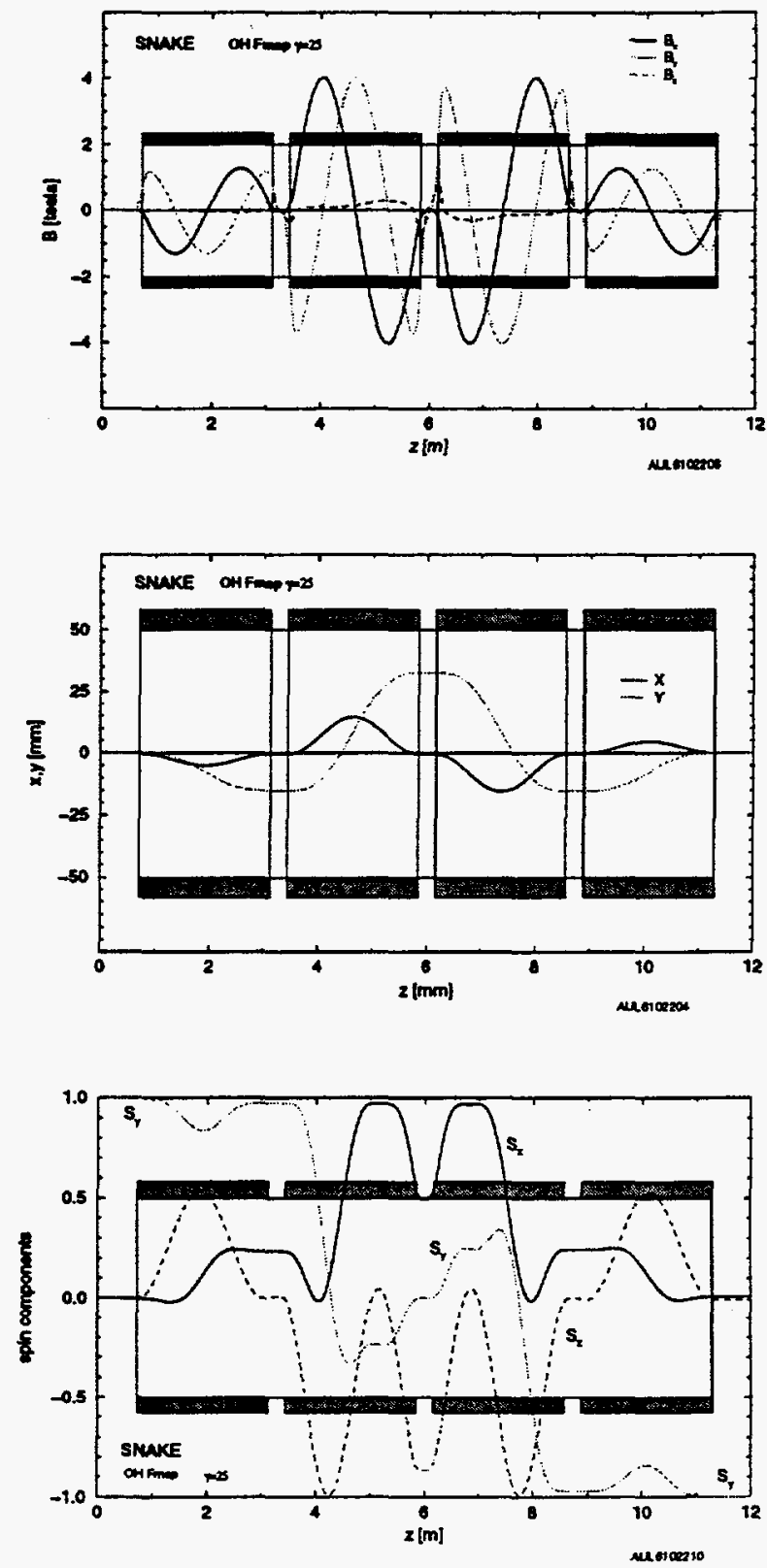

Figure 1: Magnetic field, $25 \mathrm{GeV}$ proton trajectory, and proton spin components versus distance through a RHIC Siberian Snake.

Field quality is also an issue for the helical magnets. The intrinsic twist of the helices in addition to the magnet design and construction errors generate nonlinear fields. While the nonlinear field components tend to average to zero over the length of the helical dipole, the protons follow a trajectory which is not centered within the magnet. Thus, one expects to see 
feed-down effects. For example, a sextupole component in the magnet will generate a tune shift due to the off-centered orbit. Analytical estimates indicate that the intrinsic tune shift at $25 \mathrm{GeV}$ due to two Snakes in RHIC is on the order of $\Delta v=0.015$, and that a sextupole component in the magnet design of strength $b_{2}=2 \times 10^{-4} / \mathrm{cm}^{2}$ will give approximately the same tune shift[9],[10]. Particle tracking results are in qualitative agreement with these estimates[11]. It may be possible to design the magnet cross-section with multipole moments which help compensate the intrinsic nonlinear field[12].

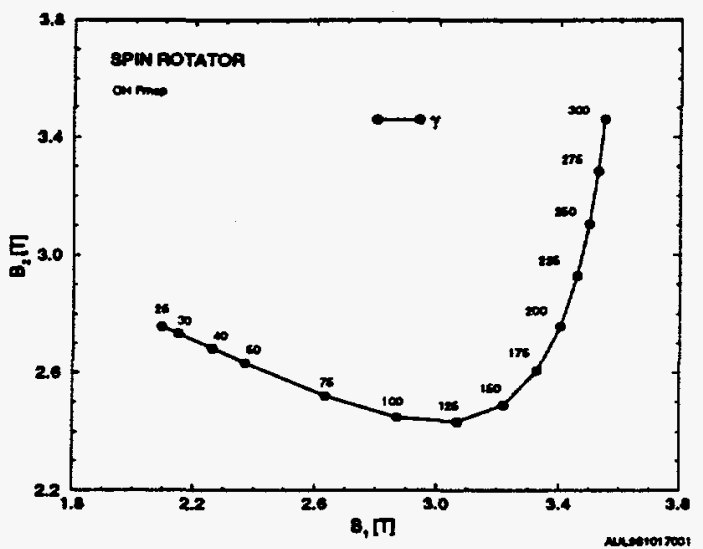

Figure 2: Spin Rotator field strengths for various RHIC beam energies.

\section{PROTOTYPE MAGNET DEVELOPMENT}

A total of 48 individual full-helical dipole magnets will be required for the project. The four magnets needed to create one Snake or one Rotator will be mounted inside of a single cryostat. The magnets will be constructed with hundreds of turns of superconducting cable to minimize both the required current and the associated heat leak through the power leads. At present, two possible techniques for producing helical coils are being investigated. (See Figure 3.) The first,

\section{Slotted Coil}
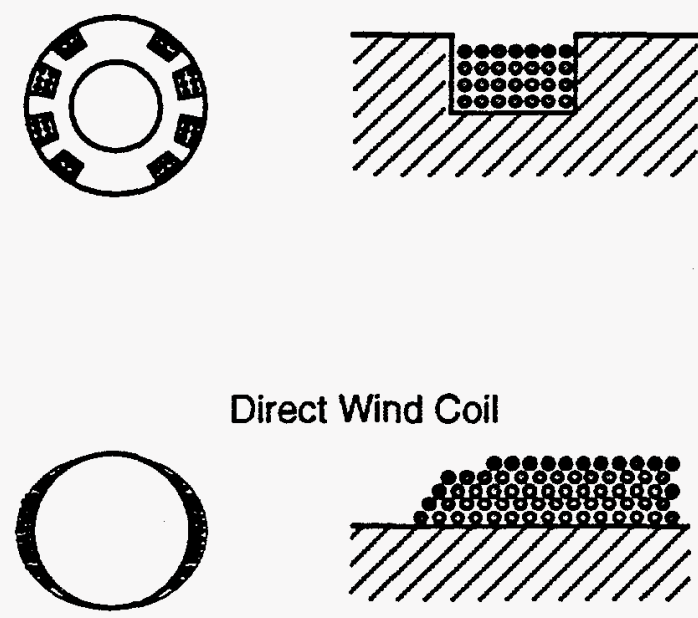

Figure 3: Possible Magnet Technologies. called the "slotted" coil method, is based upon established BNL technology used to produce the RHIC sextupole magnets. It consists of an ordered wound cable placed into helical grooves that have been milled into a thick-walled aluminum cylinder. Thin sheets of epoxy-loaded fiberglass are placed between layers, and the entire assembly is cured at elevated temperature and with radial pressure to produce a compact, strong wire matrix. The second, called the "direct-wind" coil method, consists of the same type of cable being bonded directly onto a stainless steel cylinder in multiple layers. The cable is wound into a helical pattern using a computer controlled multipleaxis winding machine. This "direct-wind" process holds the promise of a low cost production method.

Half-length prototype helical dipole magnets have been manufactured using both processes. The first prototype produced, using the direct wind method, was completed by AML, Inc., of Palm Bay, FL, and tested at BNL in November, 1996. This magnet reached 475 A (approximately $4 \mathrm{~T}$ ) at a temperature of $4.35 \mathrm{~K}$. This was the first time a low current direct-wind magnet reached this field level, the previous mark being roughly $2.5 \mathrm{~T}$. The magnet experienced significant training, which was later diagnosed as being due to voids in the cable-epoxy matrix along the first cable layer near the poles. A solution to this problem has been proposed, and a second prototype magnet is scheduled to be built in the near future.

The first complete slotted magnet prototype was constructed at BNL and tested in February, 1997. After two training quenches, the magnet plateaued at its short-sample current of $400 \mathrm{~A}$, corresponding to a field of $4.8 \mathrm{~T}$. The coil for this prototype magnet was wound by hand, and methods for automating the winding process are being investigated. Further details of each of these magnet designs and their performance can be found elsewhere in these proceedings[13],[14]. The prototype magnets have been modeled in 3-D to examine the end effects and to assist in the final magnet parameterization. Results can be found elsewhere in these proceedings[15].

\section{REFERENCES}

[1] E. D. Courant, 8th Intl. Symp. on High Energy Spin Physics, AIP Conf. Proc. 187, 1085 (1989).

[2] V. I. Ptitsin and Yu. M. Shatunov, BNL Report BNL-52453, 15 (1994).

[3] J. P. Blewett and R. Chasman, J. App. Phys. 48, $2692(1977)$.

[4] E. D. Courant, BNL AGS/RHIC/SN-010, June,

5] M. Syphers, BNL AGS/RHIC/SN-020, Feb. 1996.

6 A. Luccio, BNL AGS/RHIC/SN-042, Nov. 1996.

77 M. Syphers, BNL AGS/RHIC/SN-016, Dec. 1995

[8] M. Syphers, BNL Report, in preparation.

[9] M. Syphers, BNL AGS/RHIC/SN-015, Nov. 1995.

[10]T. Katayama, BNL AGS/RHIC/SN-038

[11]W. Fischer, BNL AGS/RHIC/SN-034, Aug. 1996.

[12]K. Tominaka, BNL AGS/RHIC/SN-054, Apr. 1997.

[13] R. Meinke, et al., these proceedings.

[14]E. Willen, et al., these proceedings.

[15] M. Okamura, et al., these proceedings. 


\section{DISCLAIMER}

This report was prepared as an account of work sponsored by an agency of the United States Government. Neither the United States Government nor any agency thereof, nor any of their employees, makes any warranty, express or implied, or assumes any legal liability or responsibility for the accuracy, completeness, or usefulness of any information, apparatus, product, or process disclosed, or represents that its use would not infringe privately owned rights. Reference herein to any specific commercial product, process, or service by trade name, trademark, manufacturer, or otherwise does not necessarily constitute or imply its endorsement, recommendation, or favoring by the United States Government or any agency thereof. The views and opinions of authors expressed herein do not necessarily state or reflect those of the United States Government or any agency thereof. 


\section{DISCLAMMER}

Portions of this document may be illegible in electronic image products. Images are produced from the best available original document. 